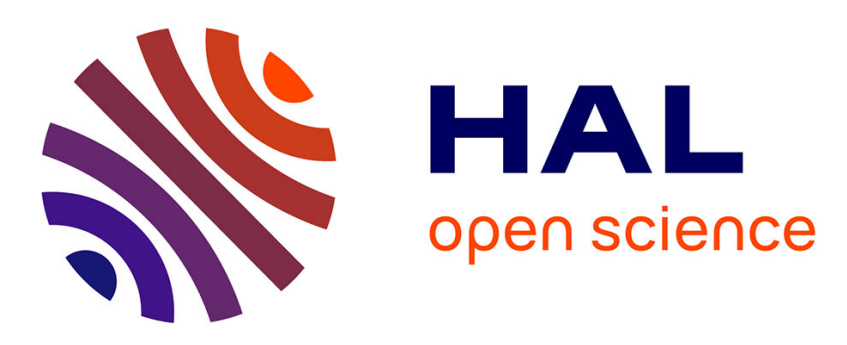

\title{
Merging conflicting propositional knowledge by similarity
}

\author{
Steven Schockaert, Henri Prade
}

\section{To cite this version:}

Steven Schockaert, Henri Prade. Merging conflicting propositional knowledge by similarity. 21st IEEE International Conference on Tools with Artificial Intelligence, Nov 2009, Newark, New Jersey, United States. pp.224-228, 10.1109/ICTAI.2009.56 . hal-03356850

\section{HAL Id: hal-03356850 https://hal.science/hal-03356850}

Submitted on 29 Sep 2021

HAL is a multi-disciplinary open access archive for the deposit and dissemination of scientific research documents, whether they are published or not. The documents may come from teaching and research institutions in France or abroad, or from public or private research centers.
L'archive ouverte pluridisciplinaire HAL, est destinée au dépôt et à la diffusion de documents scientifiques de niveau recherche, publiés ou non, émanant des établissements d'enseignement et de recherche français ou étrangers, des laboratoires publics ou privés. 


\section{Merging Conflicting Propositional Knowledge by Similarity}

\author{
Steven Schockaert* \\ Ghent University \\ Dept. of App. Math. and Comp. Sc. \\ Krijgslaan 281, 9000 Gent, Belgium \\ steven.schockaert@ugent.be
}

\author{
Henri Prade \\ Toulouse University, Univ. Paul Sabatier \\ IRIT, CNRS, 118 route de Narbonne, \\ 31062 Toulouse Cedex 09, France \\ prade@irit.fr
}

\begin{abstract}
The paper discusses a new approach to merging conflicting propositional knowledge bases which builds on the idea that consistency can often be restored by interpreting propositions more flexibly, thus enlarging their sets of models.
\end{abstract}

\section{Introduction}

Pieces of information may be conflicting, especially when they come from different sources. Obviously there may be many reasons for this. A source may e.g. provide statements that are slightly too precise w.r.t. the actual state of available information. Second, sources are often heterogeneous in their use of categories for describing reality, and they may make slightly different uses of the same label. Lastly, information may also evolve with time or space.

This suggests that information provided by a source should sometimes be understood with some flexibility. Although there has been a large number of works in information fusion (see e.g. [4]), often based on the general setting of distancebased merging (see $[5,3]$ in particular), existing approaches do not readily allow to recover consistency by explicitly enlarging the set of models corresponding to propositions. The aim of this paper is to explore the latter view of merging.

\section{Background}

Let $A$ be a finite set of atomic propositions (or atoms). The set of all models of a set of propositional formulas $K$ is denoted by $\llbracket K \rrbracket$. In the

\footnotetext{
* Postdoctoral fellow of the Research Foundation - Flanders
}

remainder of this paper, unless stated otherwise, $\mathcal{K}=\left(K_{1}, K_{2}, \ldots, K_{n}\right)$ will be a list of propositional knowledge bases, which are individually consistent, while together being inconsistent, i.e. $\forall i . \llbracket K_{i} \rrbracket \neq \emptyset$ and $\llbracket \bigcup_{i=1}^{n} K_{i} \rrbracket=\bigcap_{i=1}^{n} \llbracket K_{i} \rrbracket=\emptyset$. Given a pre-order $\left(P, \leq_{P}\right)$ and $A \subseteq P$, we let $\operatorname{Min}\left(A, \leq_{P}\right)$ denote the set of minimal elements in $A$ w.r.t. $\leq_{P}$.

The problem of interest is finding a consistent knowledge base $\Delta(\mathcal{K})$ which reflects the given knowledge bases $K_{i}$ as good as possible. In addition to $\mathcal{K}$, also a set of integrity constraints $C$ may be available that have to be satisfied in every model of $\Delta(\mathcal{K})$. Merging strategies are then specified in terms of a semimetric $d$ between interpretations. For an interpretation $I$, define $d(I, \mathcal{K})$ as

$$
d(I, \mathcal{K})=\left(\min _{J \in \llbracket K_{1} \rrbracket} d(I, J), \ldots, \min _{J \in \llbracket K_{n} \rrbracket} d(I, J)\right)
$$

Let $f$ be a nondecreasing $\left[0,+\infty\left[{ }^{n}-P\right.\right.$ mapping, where $\mathcal{P}=\left(P, \leq_{P}\right)$ is a preorder. The mapping $f$ is essentially an aggregation strategy which allows to compare the vectors $d(I, \mathcal{K})$ corresponding to different interpretations $I$. Thus, $d$ and $f$ induce a pre-order $\leq_{f}^{d}$ on interpretations, defined by $I_{1} \leq_{f}^{d} I_{2}$ iff $f\left(d\left(I_{1}, \mathcal{K}\right)\right) \leq_{P} f\left(d\left(I_{2}, \mathcal{K}\right)\right)$. Using $\leq_{f}^{d}$, the result of the merging process can be defined as $\llbracket \Delta(\mathcal{K}) \rrbracket=\operatorname{Min}\left(\llbracket C \rrbracket, \leq_{f}^{d}\right)$. A common choice is to use the Hamming distance $d_{\text {Ham }}$, given by $d_{H a m}(I, J)=|(I \backslash J) \cup(J \backslash I)|$, and to define $f$ as the sum operation [5].

\section{Motivation}

Pure logical views on information fusion usually favor a strict understanding of the underlying statements, which leads to a merging strategy based on introducing disjunctions. On the other hand, decision-oriented attitudes are more inclined 
to look for compromises, thus solving conflicts by looking for "intermediary" interpretations.

Example 1. Consider atoms oc ("tomorrow the sky will be overcast"), pc ("tomorrow the sky will be partially cloudy"), and os ("tomorrow the sky will be open"), and assume that we have access to two web services with weather forecast information, whose prediction for tomorrow is conflicting with the integrity constraint that the three atoms are jointly exhaustive and pairwise disjoint $(J E P D): K_{1}=\{o c\}$ and $K_{2}=\{o s\}$. Classical merging operators typically lead to $\Delta\left(K_{1}, K_{2}\right)=$ $\{o c \vee o s\}$. However, unless one of the sources is substantially less reliable, the most intuitive conclusion would be $\Delta\left(K_{1}, K_{2}\right)=\{p c\}$.

In this example, $p c$ acts as a borderline case of both $o s$ and $o c$, which is why the result $\Delta\left(K_{1}, K_{2}\right)=$ $\{p c\}$ seems so appealing. Given the importance of borderline cases to explain conflicts, it may be useful to introduce atoms that do not occur in any of the given knowledge bases; e.g. the result of merging $\{o s\}$ and $\{p c\}$ may be defined as an $o s-p c$ borderline case (i.e. a sky which is open, apart from a few small clouds).

Example 2. Assume that there are five different $J E P D$ atoms between os and oc, say $p c_{-2}, p c_{-1}$, $p c_{0}, p c_{1}, p c_{2}$ with easy-to-guess meanings. Now there are different outcomes that may be advocated. Adhering to a symmetry principle, and trying to be as precise as possible, one may desire $\Delta\left(K_{1}, K_{2}\right)=\left\{p c_{0}\right\}$. However, also the more cautious $\Delta\left(K_{1}, K_{2}\right)=\left\{p c_{-1} \vee p c_{0} \vee p c_{1}\right\}$ and $\Delta\left(K_{1}, K_{2}\right)=\left\{p c_{-2} \vee p c_{-1} \vee p c_{0} \vee p c_{1} \vee p c_{2}\right\}$ can intuitively be justified.

When more than two knowledge bases are available, merging strategies may implement arbitration-like behaviour, or (soft) majority principles, as illustrated in the next example.

Example 3. When a third knowledge base $K_{3}=$ $\{o s\}$ becomes available, $p c_{-2}$ and $p c_{-1}$ may be perceived as more plausible than $p c_{0}$. Thus, the desired behaviour of the merging operator might be $\Delta\left(K_{1}, K_{2}, K_{3}\right)=\left\{p c_{-2} \vee p c_{-1}\right\}$, or even $\Delta\left(K_{1}, K_{2}, K_{3}\right)=\left\{o s \vee p c_{-2} \vee p c_{-1} \vee p c_{0}\right\}$.

\section{Qualitative Similarity}

Manipulating atoms Since it is usually not possible to quantify the strength of similarities between atoms, we focus on a qualitative notion of

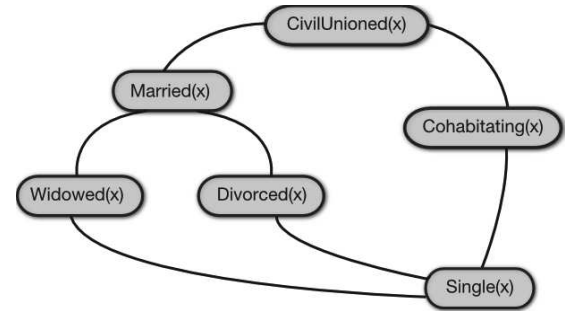

Figure 1. Similarity graph for atoms related to marriage.

similarity. For each atom $a$, we assume that a sequence of weakenings $a^{*}, a^{* *}, a^{* * *}$, etc. is available, corresponding to increasingly more tolerant interpretations of the assertion modeled by atom $a$. In general, we write $a^{(k)}$ for the $k^{t h}$ element in this sequence, and $a^{(0)}=a$. The integrity constraints $C$ then specify what is known about an atom such as $a^{(k)}$. In the same way, we assume that a sequence of tightenings $a_{*}, a_{* *}$, etc. is available, where we write $a_{(k)}$ for the $k^{t h}$ element.

In practice, such sequences of weakenings and tightenings can be defined in terms of a similarity graph $G=(A, S)$, where the set of nodes $A$ coincides with the set of atoms, and there is an edge $(a, b)$ in $S$ if atoms $a$ and $b$ are similar. Given a similarity graph $G$, we can interpret $a^{(k)}$ (resp. $\left.a_{(k)}\right)$ as the disjunction (resp. conjunction) of all atoms whose distance to $a$ in $G$ is at most $k$. As an example, Figure 1 depicts a similarity graph involving predicates related to marriage.

Weakenings and tightenings can also be applied to propositions and knowledge bases. Positive occurrences of an atom $a$ are then replaced by an atom of the form $a^{(k)}$, and negative occurrences by an atom of the form $a_{(k)}$. For instance, for $K=\{\neg p \vee q, r\}$ we have $K^{*}=\left\{\neg p_{*} \vee q^{*}, r^{*}\right\}$.

Manipulating interpretations Using the similarity information encoded by the sequences of weakenings $a^{(k)}$, we can define a sequence of expansions of an interpretation $I$ as follows. Let $C_{\text {sim }}$ be the fragment of the integrity constraints in which the semantics of the atoms $a^{(k)}$ and $a_{(k)}$ is specified. Then we define the $k$-expansion of an interpretation $I$ (where $a \in I$ means $I \models a$ ) as

$$
\langle I\rangle^{k}=\left\{b \mid \exists a \in I . C_{\text {sim }} \cup\{b\} \models a^{(k)}\right\}
$$

Note that by definition of $C_{\text {sim }}, C_{\text {sim }} \cup\{b\} \models a^{(k)}$ intuitively means that $b$ is similar to $a$. We write $\langle I\rangle$ for $\langle I\rangle^{1}$. Intuitively, $\langle I\rangle$ is the set of atoms that 
are plausible, given that the atoms in $I$ are asserted. It can be verified that for every $I$ in $\llbracket C \rrbracket$, it holds that $I \in \llbracket a^{*} \rrbracket$ iff $I \cap\langle\{a\}\rangle \neq \emptyset$. Similarly, we define the $k$-contraction $[I]^{k}$ of $I$ as the set of atoms from $I$ that are not similar to any atoms outside $I$, viz. $[I]^{k}=A \backslash\langle A \backslash I\rangle^{k}$.

\section{Semantic Merging Operators}

Approximate covers When the given knowledge bases $K_{1}, \ldots, K_{n}$ are not conflicting, the result of the merging process is such that

$$
\llbracket \Delta(\mathcal{K}) \rrbracket=\llbracket C \rrbracket \cap \bigcap_{i=1}^{n} \llbracket K_{i} \rrbracket \neq \emptyset
$$

In other words, $I \in \llbracket \Delta(\mathcal{K}) \rrbracket$ iff $I \in \llbracket C \rrbracket$ and there are $J_{i} \in \llbracket K_{i} \rrbracket$ such that $I=J_{1}=\ldots=J_{n}$. This latter criterium can be split into the following criteria, viewing interpretations as sets of atoms:

$$
I \subseteq J_{i} \quad J_{i} \subseteq I
$$

When the knowledge bases are conflicting, no interpretation $I$ can be found satisfying (2) for all $i \in\{1, \ldots, n\}$. In such a case, the criteria can be weakened using expansions or contractions:

$$
\begin{aligned}
I \subseteq\left\langle J_{i}\right\rangle^{\alpha_{i}} & \text { (3) } & {[I]^{\alpha_{i}^{\prime}} \subseteq J_{i} } \\
J_{i} \subseteq\langle I\rangle^{\beta_{i}} & \text { (4) } & {\left[J_{i}\right]^{\beta_{i}^{\prime}} \subseteq I }
\end{aligned}
$$

First, (3) and (4) ensure that every atom in $I$ is similar to an atom in $J_{i}$, and vice versa. Next, (5) and (6) ensure that $I$ should include all atoms from $J_{i}$ that are not borderline cases, and vice versa. It is easy to show that (3)-(6) also writes as

$$
\begin{aligned}
& {\left[\operatorname{coJ}_{i}\right]^{\alpha_{i}} \subseteq \operatorname{coI} \quad(7) \quad \operatorname{coJ} J_{i} \subseteq\langle\operatorname{coI}\rangle^{\alpha_{i}^{\prime}}} \\
& {[c o I]^{\beta_{i}} \subseteq \operatorname{coJ}_{i} \quad(8) \quad \operatorname{coI} \subseteq\left\langle\operatorname{coJ}_{i}\right\rangle^{\beta_{i}^{\prime}}}
\end{aligned}
$$

where the complement $c o I=A \backslash I$. Thus we have the important property that positive (e.g. $a \in I$ ) and negative (e.g. $a \notin I$ ) information is treated in the same way.

An interpretation $I \in \llbracket C \rrbracket$ is called an approximate cover of $\mathcal{K}$ w.r.t. the weight vector $\left(\alpha_{1}, \ldots, \alpha_{n}, \beta_{1}, \ldots, \beta_{n}, \alpha_{1}^{\prime}, \ldots, \alpha_{n}^{\prime}, \beta_{1}^{\prime}, \ldots, \beta_{n}^{\prime}\right), \quad$ if there are models $J_{i}$ of each $K_{i}$ such that (3)-(6) are satisfied for all $i$ in $\{1, \ldots, n\}$. The smaller the coefficients $\alpha_{i}, \beta_{i}, \alpha_{i}^{\prime}, \beta_{i}^{\prime}$, the more "compatible" $I$ will be to each of the knowledge bases. An approximate cover w.r.t. weight vector $\mathbf{x}$ will also be called an $\mathbf{x}$-cover. Throughout this paper, we tacitly assume that there is a $k \in \mathbb{N}$, such that $\mathcal{K}$ has at least one $\mathbf{k}$-cover with $\mathbf{k}=(k, \ldots, k)$. If no such $k$ exists (or if $k$ is too high), $\mathcal{K}$ contains conflicts that cannot (realistically) be resolved by means of similarity (e.g. accidental errors), and the proposed merging strategy needs to be combined with a classical merging strategy.

Now, let $W_{\mathbf{x}}$ be the set of $\mathbf{x}$-covers of $\mathcal{K}$, and assume that a certain aggregation strategy $f$ is available to compare the weight vectors, i.e. let $f$ be an $\mathbb{N}^{4 n}-P$ mapping for some pre-order $\left(P, \leq_{P}\right)$. Then $f$ naturally induces a pre-order $\leq_{f}$ on interpretations from $W_{\mathbf{k}}$ such that $I_{1} \leq_{f} I_{2}$ iff

$$
\begin{aligned}
& \forall \mathbf{w}_{\mathbf{2}} \in \mathbb{N}^{4 n} \cdot I_{2} \in W_{\mathbf{w}_{\mathbf{2}}} \\
& \quad \Rightarrow \exists \mathbf{w}_{\mathbf{1}} \in \mathbb{N}^{4 n} \cdot f\left(w_{1}\right) \leq_{P} f\left(w_{2}\right) \wedge I_{1} \in W_{\mathbf{w}_{\mathbf{1}}}
\end{aligned}
$$

Typical examples for $f$ are the sum, the sum of squares, the maximum, as well as the leximax and Pareto strategies. In each case, it seems desirable to impose that models of $\Delta(\mathcal{K})$ are as compatible to the given knowledge bases as possible:

$$
\llbracket \Delta(\mathcal{K}) \rrbracket \subseteq \operatorname{Min}\left(W_{\mathbf{k}}, \leq_{f}\right)
$$

Parsimonious covers There is a clear analogy between the preceding discussion and the problem of causal diagnosis, in the sense that a diagnosis should cover the observed symptoms. To stress this analogy, throughout this paper, we borrow some terminology from the parsimonious covering theory [7].

A merging operator could be obtained by replacing the subset relation in (11) by an equality. Intuitively, however, such a merging operator would be too liberal, as it could lead to atoms being replaced by similar atoms, even when this is not required to obtain consistency.

Example 4. Consider the similarity graph $G$ from Figure 1, and let

$$
\begin{aligned}
& K_{1}=\{M a(j o h n), M a(b o b)\} \\
& K_{2}=\{C o(j o h n), M a(b o b)\}
\end{aligned}
$$

where the predicates from Figure 1 are abbreviated by their first two letters. Assume furthermore that the integrity constraints $C$ specify that the six predicates from Figure 1 are JEPD. Now, consider the merging operator that results from replacing the subset relation in (11) by an equality, and choosing $f=\max$. It can be verified that this leads to

$$
\begin{aligned}
\Delta(\mathcal{K})=\{C i(j \circ h n), & M a(b \circ b) \vee C i(b o b) \\
& \vee D i(b o b) \vee W i(b o b)\}
\end{aligned}
$$


which is clearly too cautious: there is no reason to doubt that bob is Married.

Let $\mathcal{J}=\left(J_{1}, \ldots, J_{n}\right)$ be models of $K_{1}, \ldots, K_{n}$. Our approach is based on the observation that $I$ and $J=\bigcup_{i=1}^{n} J_{i}$ can be partitioned in $k+1$ disjoint subsets, resp. $I^{\mathcal{J}, l}$ and $J^{I, l}(l \in\{0, \ldots, k\})$ as follows.

$$
\begin{aligned}
& \left.I^{\mathcal{J}, l}=I \cap \bigcap_{i=1}^{n}\left\langle J_{i}\right\rangle^{l} \backslash \bigcap_{i=1}^{n}\left\langle J_{i}\right\rangle^{l-1}\right) \\
& J^{I, l}=J \cap\left(\langle I\rangle^{l} \backslash\langle I\rangle^{l-1}\right)
\end{aligned}
$$

if $l>0$, while $I^{\mathcal{J}, 0}=I \cap\left(\bigcap_{i=1}^{n} J_{i}\right)$ and $J^{I, 0}=$ $J \cap I$. For $l>0, I^{\mathcal{J}, l}$ contains those atoms that are not compatible with all knowledge bases. Moreover, the larger the value of $l$, the larger the degree of incompatibility. In the same way, for $l>0$, $J^{I, l}$ contains the atoms in the considered interpretations of the knowledge bases that are not equal, albeit still somewhat similar, to atoms in $I$. To maintain the symmetry between positive and negative information, in entirely the same way we also partition $I_{c o}=c o I$ and $J_{c o}=\bigcup_{i=1}^{n} c o J_{i}$ into subsets $I_{c o}^{\mathcal{J}, l}$ and $J_{c o}^{I, l}$. Now each of the four partitions $I^{\mathcal{J}, l}, J^{I, l}, I_{c o}^{\mathcal{J}, l}$ and $J_{c o}^{I, l}$ induces a pre-order, resp. $\leq_{1}, \leq_{2}, \leq_{3}$ and $\leq_{4}$, which is a lexicographic extension of the subset relation. Namely, we have $I_{1} \leq_{1} \quad I_{2}$ iff $I_{1}=I_{2}$ or there is a choice of models $\mathcal{J}=\left(J_{1}, \ldots, J_{n}\right)$ such that for each choice of models $\mathcal{J}^{\prime}=\left(J_{1}^{\prime}, \ldots, J_{n}^{\prime}\right)$ it holds that

$$
\exists l>0 . I_{1}^{\mathcal{J}, l} \subset I_{2}^{\mathcal{J}^{\prime}, l} \wedge \forall m>l . I_{1}^{\mathcal{J}, m}=I_{2}^{\mathcal{J}^{\prime}, m}
$$

The definitions of $\leq_{2}, \leq_{3}$ and $\leq_{4}$ are analogous. An interpretation $I$ is called an $\mathrm{x}$-irredundant cover of $\mathcal{K}$ if $I$ is an $\mathbf{x}$-cover, and $I$ is Pareto optimal among all $\mathbf{x}$-covers, w.r.t. $\leq_{1}, \leq_{2}, \leq_{3}$ and $\leq_{4}$. If we write $X_{\mathrm{x}}$ for the set of all $\mathrm{x}$-irredundant covers, the preceding discussion leads to the following requirement:

$$
\llbracket \Delta(\mathcal{K}) \rrbracket \subseteq \operatorname{Min}\left(X_{\mathbf{k}}, \leq_{f}\right)
$$

Note that when changing the subset relation in (12) into an equality, the resulting merging operator indeed leads to $\Delta(\mathcal{K})=\{C i($ john $), M a(b o b)\}$ in the case of Example 4.

Possibilistic approach Consider again the situation from Example 3, and assume that the merging operator is defined by replacing the subset relation in (12) by an equality. Depending on the choice of the aggregation function $f$, we obtain $\Delta(\mathcal{K})=$ $\left\{p c_{0}\right\}$, for $f=\max , \Delta(\mathcal{K})=\{o s\}$, for $f=\sum$, $\Delta(\mathcal{K})=\left\{o s \vee p c_{-2} \vee p c_{-1} \vee p c_{0} \vee p c_{1} \vee p c_{2} \vee o c\right\}$ for $f$ the Pareto ordering, and $\Delta(\mathcal{K})=\left\{p c_{-1}\right\}$ for $f$ the sum of squares, the latter offering a more subtle balance between the different sources. Clearly, the Pareto ordering is too cautious as its result is entailed even by the integrity constraints $C$, whereas the result of each of the other operators is too specific, as witnessed by the fact that each choice leads to a different result. An appealing solution is to define the result of the merging process as a possibilistic logic base, which encodes different conclusions with different levels of certainty; for instance, when implementing a soft majority behavior, a desirable result of the merging operator would be

$$
\begin{aligned}
& \Delta(\mathcal{K})=\left\{\left(p c_{-1}, \lambda_{1}\right),\left(p c_{-2} \vee p c_{-1} \vee p c_{0}, \lambda_{2}\right),\right. \\
& \left(o s \vee p c_{-2} \vee p c_{-1} \vee p c_{0} \vee p c_{1}, \lambda_{3}\right), \\
& \left(o s \vee p c_{-2} \vee p c_{-1} \vee p c_{0} \vee p c_{1} \vee p c_{2}, \lambda_{4}\right), \\
& \left.\left(o s \vee p c_{-2} \vee p c_{-1} \vee p c_{0} \vee p c_{1} \vee p c_{2} \vee o c, 1\right)\right\}
\end{aligned}
$$

for some $0<\lambda_{1}<\lambda_{2}<\lambda_{3}<\lambda_{4}<1$; note that only the relative ordering of the certainty levels is important. At the semantic level, this approach amounts to specifying a degree of possibility (or compatibility) for each interpretation $I$, rather than selecting a single set of optimal interpretations. Assume, for simplicity, that the preorder $\left(P, \leq_{P}\right)$ which is used to compare weight vectors is a total order. Then there exists a mapping $\phi$ from $W_{\mathbf{k}}$ to $[0,1]$ such that $\phi(I)=1$ iff $I \in \operatorname{Min}\left(W_{\mathbf{k}}, \leq_{f}\right)$, and $\phi\left(I_{1}\right) \leq \phi\left(I_{2}\right)$ iff for each $\mathrm{x}_{1}$ such that $I_{1}$ is an $\mathbf{x}_{1}$-cover, there is an $\mathbf{x}_{2}$ such that $I_{2}$ is an $\mathbf{x}_{\mathbf{2}}$-cover and $f\left(\mathbf{x}_{\mathbf{2}}\right) \leq_{P} f\left(\mathbf{x}_{\mathbf{1}}\right)$. Using $\phi(I)$ as the possibility degree of interpretation $I$, if $I \in W_{\mathbf{k}}$, and 0 otherwise, we obtain a solution equivalent to (13) when using the sum of squares for $f$.

\section{Syntactic Merging Operators}

In this section, we propose a syntactically defined merging operator, which uses the technique for weakening propositions from Section 4. First, each of the propositional knowledge bases $K_{i}$ is converted into a possibilistic knowledge base $K_{i}^{\text {pos }}$ as follows

$$
\begin{aligned}
K_{i}^{\text {pos }}= & \left\{\left(\gamma, \lambda_{0}\right) \mid \gamma \in K_{i}\right\} \cup\left\{\left(\gamma^{*}, \lambda_{1}\right) \mid \gamma \in K_{i}\right\} \\
& \cup \ldots \cup\left\{\left(\gamma^{(k)}, \lambda_{k}\right) \mid \gamma \in K_{i}\right\}
\end{aligned}
$$

where $0 \leq \lambda_{0}<\ldots<\lambda_{k-1}<\lambda_{k}=1$. Note how more liberal interpretations receive a higher 
certainty level. For example, the knowledge base $K_{1}=\{\neg p \vee q, r\}$ is changed into $(k=2)$ :

$$
\begin{aligned}
K_{1}^{p o s}=\{( & \left(\neg p \vee q, \lambda_{0}\right),\left(r, \lambda_{0}\right),\left(\neg p_{*} \vee q^{*}, \lambda_{1}\right), \\
& \left.\left(r^{*}, \lambda_{1}\right),\left(\neg p_{* *} \vee q^{* *}, 1\right),\left(r^{* *}, 1\right)\right\}
\end{aligned}
$$

Furthermore, as the integrity constraints $C$ are certain, we define its possibilistic counterpart $C^{\text {pos }}$ as

$$
C^{p o s}=\{(\gamma, 1) \mid \gamma \in C\}
$$

Now let $\mathcal{K}^{\text {pos }}=K_{1}^{\text {pos }} \cup \ldots \cup K_{n}^{\text {pos }} \cup C^{\text {pos }}$. To define the merged knowledge base, we calculate the inconsistency level ${ }^{1}$ of $\mathcal{K}^{\text {pos }}$. If $\operatorname{inc}\left(\mathcal{K}^{\text {pos }}\right)=0$, the knowledge bases in $\mathcal{K}$ are consistent, and the result of the fusion ${ }^{2}$ is $\Delta(\mathcal{K})=\left(\mathcal{K}^{\text {pos }}\right)_{\underline{0}}$, which is equivalent to $K_{1} \cup \ldots \cup K_{n} \cup C$. In general, the result of the fusion is defined as $\Delta(\mathcal{K})=\left(\mathcal{K}^{\text {pos }}\right)_{\text {inc }\left(\mathcal{K}^{\text {pos }}\right)}$. Adapting this method to produce graded results such as (13) is straightforward: simply remove all propositions from $\mathcal{K}^{\text {pos }}$ with a certainty level which is less than or equal to $i n c\left(\mathcal{K}^{p o s}\right)$. The following proposition clarifies the link with the semantic approach from Section 5.

Proposition 1. For each atom $a$, let $a^{(l)}$ and $a_{(l)}$ be defined in terms of a similarity graph $G$. It holds that

$$
\begin{aligned}
\llbracket\left(\mathcal{K}^{\text {pos }}\right)_{\lambda_{l}} \rrbracket=\{I \in \llbracket C \rrbracket \mid \forall i \in\{1, \ldots, n\} . \\
\left.\exists J_{i} \in \llbracket K_{i} \rrbracket . J_{i} \subseteq\langle I\rangle^{l} \wedge \operatorname{coJ}_{i} \subseteq\langle c o I\rangle^{l}\right\}
\end{aligned}
$$

Note that models of $\left(\mathcal{K}^{\text {pos }}\right)_{\lambda_{l}}$ do not necessarily satisfy (3) and (6). This will be the case when one knowledge base states that some literal is true, whereas another one states that it, and everything similar to it, is false.

\section{Related Work}

It seems that there are very little works that look at restoring consistency in logical settings by explicitly enlarging the set of models of propositions. In this spirit, let us however mention the note [8] where the result of the revision of knowledge base $K$ by a formula $\phi$ is defined as the set of models of $\phi$ maximally similar to models of $K$. Next, the notion of expansion and contraction of interpretations is related to the use of morphological operators for fusion, which has been proposed in [2]. In the setting of description logic, the authors of [6]

\footnotetext{
${ }^{1}$ inc $(K)=\max \left\{\alpha \mid K_{\alpha}\right.$ inconsistent $\}$

${ }^{2}$ Recall that $\left(\mathcal{K}^{\text {pos }}\right)_{\underline{\alpha}}=\left\{(p, \lambda) \in \mathcal{K}^{\text {pos }} \mid \lambda>\alpha\right\}$
}

look for overgeneralized concepts that create inconsistency problems, and tighten or enlarge such concepts in order to restore subconcept relations in a consistent way. Besides, it has been shown [1] that possibilistic logic can be used to syntactically encode distance-based merging operators. As indicated above, it is also useful for similarity-based merging.

\section{Conclusions}

We have explored a novel approach to information fusion, stemming from the observation that inconsistencies often arise because propositions are interpreted too strict. Using a number of motivating examples, we have advocated that natural repairs can be obtained by using a notion of similarity between different atoms. Subsequently, we have discussed the criteria that should ideally be satisfied by models of the merged knowledge base. We have emphasized the trade-off that arises between having cautious and precise conclusions, and we proposed an encoding in possibilistic logic to cope with this. Finally, we have illustrated how a syntactic counterpart to the semantically defined merging operators can be obtained.

\section{References}

[1] S. Benferhat, D. Dubois, S. Kaci, and H. Prade. Possibilistic merging and distance-based fusion of propositional information. Annals of Math. and Artif. Intelligence, 34(1-3):217-252, 2002.

[2] I. Bloch and J. Lang. Towards mathematical morpho-logics. In Proc. IPMU, pages 1405-1412, 2000.

[3] S. Konieczny, J. Lang, and P. Marquis. Distancebased merging: a general framework and some complexity results. In Proc. KR'02, pages 97-108, 2002.

[4] L. Cholvy and A. Hunter, editors. Special Issue on Data and Knowledge Fusion (Parts I \& II), volume 16 of Intern. J. of Intelligent Systems. 2001.

[5] J. Lin. Integration of weighted knowledge bases. Artificial Intelligence, 83(2):363 - 378, 1996.

[6] E. Ovchinnikova, T. Wandmacher, and K.-U. Kühnberger. Solving terminological inconsistency problems in ontology design. Interoperability in Business Information Systems, 2(1):65-80, 2007.

[7] J. Reggia, D. Nau, P. Wang, and H. Peng. A formal model of diagnostic inference. Information Sciences, 37:227-285, 1985.

[8] R. Rodriguez, P. Garcia, and L. Godo. Relating similarity-based models, counterfactuals and theory change. In Proc. EUFIT'95, pages 230-234, 1995. 